\title{
Calculation of Dynamical and Many-Body Observables with the Polynomial Expansion Method for Spin-Fermion Models
}

\author{
G. Alvarez and T. C. Schulthess \\ Computer Science 85 Mathematics Division, Oak Ridge National Laboratory, Oak Ridge, TN 37831
}

\begin{abstract}
The calculation of two- and four-particle observables is addressed within the framework of the truncated polynomial expansion method (TPEM). The TPEM replaces the exact diagonalization of the one-electron sector in models for fermions coupled to classical fields such as those used in manganites and diluted magnetic semiconductors. The computational cost of the algorithm is $O(N)$ - with $N$ the number of lattice sites - for the TPEM which should be contrasted with the computational cost of the diagonalization technique that scales as $O\left(N^{4}\right)$. By means of the TPEM, the density of states, spectral function and optical conductivity of a double-exchange model for manganites are calculated on large lattices and compared to previous results and experimental measurements. The ferromagnetic metal becomes an insulator by increasing the direct exchange coupling that competes with the double exchange mechanism. This metal-insulator transition is investigated in three dimensions.
\end{abstract}

PACS numbers: 75.10.-b,71.10.Fd,02.70.Lq

\section{INTRODUCTION}

Strongly correlated electron systems continue to be one the most important areas of research in condensed matter physics. In this context, spin-fermion models, $i$. $e$. fermion systems coupled to classical fields, provide a bridge between the full Hubbard model and models with only classical degrees of freedom. The later fail to capture important physical aspects, like carrier mediated ferromagnetism in manganites while the former are usually too complex to study without approximations due to the sign problem 1

Spin-fermion models contain all the physical properties of a strongly correlated electron system, including the effect of the carriers and inhomogeneous local charge density. They have been successful in the description of many materials, e. g. manganites ${ }^{2}$ and diluted magnetic semiconductors (DMS) $\stackrel{\underline{3}}{\underline{3}}$ Inhomogeneous phases in these materials have been found to be of importance in explaining many physical properties. It was also found that mean field theories are insufficient to obtain these phases in DMS systems 4.5 but that information from these models must be extracted without approximations. In 2005, a spin-fermion model for multiferroic materials was also studied with the diagonalization method $\underline{\underline{6}}$

The traditional method to calculate observables has been to integrate the fermions exactly at a finite temperature for every configuration of classical fields and then integrate these fields by means of a Monte Carlo procedure. Although this method - which will be referred to as DIAG - is accurate and has lead to many advances, e. $g$. the theoretical observation of phase separation in manganites ${ }^{2}$ and clustered states in DMS,,$\frac{3}{=}$ it has limitations. In fact, the largest systems that can be studied with this procedure are lattices with less than approximately 300 sites.

In 1999, Furukawa and coworkers 7 introduced a polynomial expansion of the fermionic density of states as a way of avoiding the diagonalization of the fermionic sector. This polynomial expansion method (PEM) scales as $O\left(N^{3}\right)$ as opposed to the diagonalization method that scales as $O\left(N^{4}\right), N$ being the size of the system. The method could also be parallelized, which is practically impossible for the diagonalization method. In 2003, the same researchers ${ }^{8}$ developed a truncated version of the PEM, that will be abbreviated by TPEM that scales linearly, i. e. $O(N)$, with the size of the system. All these methods do not introduce systematic errors or uncontrolled approximations and the results obtained converge to the exact result as the expansion cut-off tends to infinity.

Although the TPEM is becoming a standard way to avoid the diagonalization, $, 9.10,11.12$ observables that involve two- and four- fermionic operators have not been studied systematically. Here, it will be shown how to efficiently calculate these observables and the formalism is applied to a double exchange model for manganites. The emphasis will be on the calculation of the spectral function, $A(k, \omega)$, and optical conductivity, $\sigma(\omega)$, although the treatment applies to other observables as well.

Another algorithm that improves the integration of the one-electron sector in double exchange models is the "Hybrid Monte Carlo" (HMC) algorithm, 13 This method uses the path integral representation, introducing imaginary time and a HMC procedure to evolve the system. The TPEM works better than the HMC at low temperatures where the HMC presents increasing computational cost due to the time discretization. Another reason why we have preferred the TPEM is that the HMC method is applicable when the bands are connected and do not extend over a wide range of energies, as is the case of finite coupling systems. The TPEM also allows for easy parallelization, improving the performance even more.

The contents of this paper is the following. In a review of the TPEM for spin fermion models as introduced originally by Furukawa et al. is presented. In IIB IIC the formalism to calculate two-particle observ- 
ables is discussed as well as examples and in particular the formula for the spectral function, $A(k, \omega)$. In the formalism is extended to include four-particle observables and to allow for the calculation of the optical conductivity. The parallelization of the algorithm and its complexity is discussed in these observables are calculated for a model of manganites. The metal to insulator transition is studied in three dimensions. Finally, in Section IV we present the most important conclusions and discuss further uses of these techniques.

\section{THEORY}

\section{A. Review of the TPEM}

The brief review presented here follows closely Reference 14. Consider a model defined by a general Hamiltonian, $\hat{H}=\sum_{i j \alpha \beta} c_{i \alpha}^{\dagger} H_{i \alpha, j \beta}(\phi) c_{j \beta}$, where the indices $i$ and $j$ denote a spatial index and $\alpha$ and $\beta$ are internal degree(s) of freedom, e. g. spin and/or orbital. The Hamiltonian matrix depends on the configuration of one or more classical fields, represented by $\phi$. Although no explicit indices will be used, the field(s) $\phi$ are supposed to have a spatial dependence. The partition function for this Hamiltonian is given by: $Z=\int d \phi \sum_{n}\langle n| \exp (-\beta \hat{H}(\phi)+$ $\beta \mu \hat{N})|n\rangle$ where $|n\rangle$ are the eigenvectors of the oneelectron sector. Here $\beta=1 /\left(k_{B} T\right)$ is the inverse temperature. The number of particles (operator $\hat{N}$ ) is adjusted via the chemical potential $\mu$. The procedure to calculate observables (energy, density, action, etc) is the following. First, an arbitrary configuration of classical fields $\phi$ is selected as a starting point. The Boltzmann weight or action of that configuration, $S(\phi)$, is calculated by diagonalizing the one-electron sector at finite temperature. Then a small local change of the field configuration is proposed, so that the new configuration is denoted by $\phi^{\prime}$ and its action is evaluated to obtain the difference in action $\Delta S=S\left(\phi^{\prime}\right)-S(\phi)$. Finally, this new configuration is accepted or rejected based on a Monte Carlo algorithm like Metropolis or heat bath and the cycle starts again. In summary, the observables are traditionally obtained using exact diagonalization of the one-electron sector for every classical field configuration and Monte Carlo integration for those classical fields 2 . The PEM/TPEM replaces the diagonalization for a polynomial expansion and is briefly described here but further details can be found in Ref. 7, 8, 15. These definitions will be helpful later in deriving formulas for many-body observables.

It will be assumed that the Hamiltonian $H(\phi)$ is normalized, which simply implies a re-scaling:

$$
\begin{aligned}
H & \rightarrow(H-b) / a \\
a & =\left(E_{\max }-E_{\min }\right) / 2 \\
b & =\left(E_{\max }+E_{\min }\right) / 2,
\end{aligned}
$$

where $E_{\max }$ and $E_{\min }$ are the maximum and minimum eigenvalues of the original Hamiltonian respectively. This in turn implies that the normalized Hamiltonian has eigenvalues $\epsilon_{v} \in[-1,1]$.

Observables can be divided in three categories: (i) those that do not depend directly on fermionic operators, e. g. the magnetization, susceptibility and classical spin-spin correlations in the double exchange model, (ii) those for which a function $F(x)$ exists such that they can be written as:

$$
A(\phi)=\int_{-1}^{1} F(x) D(\phi, x) d x,
$$

where $D(\phi, \epsilon)=\sum_{\nu} \delta\left(\epsilon(\phi)-\epsilon_{\nu}\right)$, and $\epsilon_{\nu}$ are the eigenvalues of $H(\phi)$, i. e. $D(\phi, x)$ is the density of states of the system. Finally, category (iii) would include fermionic observables that cannot be expressed as in Eq. (2), in particular two and four-particle observables. In this paper the emphasis will be on this last category, that will be analyzed in the remaining sections, since dynamical observables are expressed in terms of two-particle observables, e. g. the spectral function and four-particle linear responses, e. g. optical conductivity, that had not been studied before with the TPEM or on large systems.

For category (i), the calculation is straightforward and simply involves an average over Monte Carlo configurations. Category (ii) includes the effective action or generalized Boltzmann weight and this quantity is particularly important because it is calculated very frequently throughout the Monte Carlo procedure to integrate the classical fields. Therefore, we first briefly review the calculation of these types of observables. As Furukawa et $a l$. showed, it is convenient to expand a function $F(x)$ in terms of Chebyshev polynomials in the following way:

$$
F(x)=\sum_{m=0}^{\infty} f_{m} T_{m}(x),
$$

where $T_{m}(x)$ is the $m$-th Chebyshev polynomial evaluated at $x$. Let $\alpha_{m}=2-\delta_{m, 0}$. The coefficients $f_{m}$ are calculated with the formula:

$$
f_{m}=\int_{-1}^{1} \alpha_{m} F(x) T_{m}(x) /\left(\pi \sqrt{1-x^{2}}\right) .
$$

The moments of the density of states are defined by: $\mu_{m}(\phi) \equiv \sum_{\nu=1}^{N_{\text {dim }}}\left\langle\nu\left|T_{m}(H(\phi))\right| \nu\right\rangle$, where $N_{\text {dim }}$ is the dimension of the one-electron sector. Then, the observable corresponding to the function $F(x)$, can be calculated by: $A(\phi)=\sum_{m} f_{m} \mu_{m}(\phi)$. In practice, the sum in this equation is performed up to a certain cutoff value $M$, without appreciable loss in accuracy,$\frac{7.15}{7}$ The calculation of $\mu_{m}$ is carried out recursively. $|\nu ; m\rangle=T_{m}(H)|\nu\rangle=2 H \mid \nu ; m-$ $1\rangle-|\nu ; m-2\rangle$ and hence $\mu_{2 m}=\sum_{\nu}(\langle m ; \nu \mid \nu ; m\rangle-1)$ and $\mu_{2 m+1}=\sum_{\nu}(\langle m ; \nu \mid \nu ; m+1\rangle-\langle\nu ; 0 \mid \nu ; 1\rangle)$, are used to calculate the moments. The process involves a sparse matrix-vector product, e. g. in $T_{m}(H)|\nu\rangle$, which, when considering the trace operation, yields a cost of $O\left(N^{2}\right)$ for each configuration, implying $O\left(N^{3}\right)$ for each Monte 
Carlo step. In addition, an improvement of the present method has been proposed 8 based on a truncation of the matrix-vector product mentioned before and it turns out that the resulting algorithm has a complexity linear in $N$. This approximation is controlled by the small parameter $\epsilon_{p r}$.

The function corresponding to the effective action for a configuration $\phi$ is defined by $F^{S}(x)=-\log (1+$ $\exp (-\beta(x-\mu)))$ and $S(\phi)$ admits an expansion as before with coefficients $f_{m}^{S}$ corresponding to $F^{S}(x)$. This observable is calculated very frequently in the Monte Carlo integration procedure and so it is important to calculate it efficiently. In actuality, only the difference in action, $\Delta S=S\left(\phi^{\prime}\right)-S(\phi)$ has to be computed at every change of classical fields. The authors of Ref. 8 have also developed a truncation procedure for this trace operation controlled by a small parameter, $\epsilon_{t r}$ and the current value of the effective action can be calculated faster using the moments of the expansion from the previous MC update. This truncation is based on the observation that if $\phi$ and $\phi^{\prime}$ differ only in very few sites (as is the case with local Monte Carlo updates) then the corresponding moments will differ only for certain indices. More details can be found in Ref. 8 .

In what follows, the size of the Hilbert space will be denoted by $N_{d i m}$ and it will depend on the size of the lattice as well as on the particular model to be studied. For a one-orbital double exchange model on a lattice of $N$ sites and finite coupling, $N_{\text {dim }}=2 N$; the factor of 2 is due to the spin degree of freedom.

\section{B. Density-Of-States}

The fermionic density of states of the system for a configuration of classical fields $\phi$ is given by $N_{\phi}(\omega)=$ $\sum_{\lambda} \delta\left(\omega^{\prime}-\epsilon_{\lambda}\right)$, where $\omega^{\prime}=(\omega-b) / a$. Then, the corresponding function for $D_{\phi}(\omega)$ in Eq. (2) is $F(x)=\delta\left(\omega^{\prime}-x\right)$ and the corresponding fixed coefficients are calculated from Eq. (41). The end result is

$$
N_{\phi}(\omega)=\frac{\sum_{m} \alpha_{m} T_{m}\left(\omega^{\prime}\right) \mu_{m}(\phi)}{\pi \sqrt{1-\omega^{\prime 2}}} .
$$

A Monte Carlo average over classical fields is generally needed to obtain the fermionic density of states, $i$. e., $N(\omega)=\left\langle N_{\phi}(\omega)\right\rangle_{\phi}$, and for that it suffices to calculate $\left\langle\mu_{m}(\phi)\right\rangle_{\phi}$, as can be deduced from the form of the last equation.

The sum in Eq. (5) is truncated to a certain cut-off $M$ (see, e. g. Ref. 15) and such an abrupt truncation generally results in unwanted Gibbs oscillations. Silver et al. have considered ${ }^{16.17}$ instead smooth truncations, where the moments are multiplied by damping factors, $g_{m}$. A possible choice of the damping factors, $g_{m}$, can be found in Ref. 16. 17.

\section{Two-particle observables}

The previous derivations can be slightly modified to allow for the calculation of two-particle observables. These can be written as:

$$
A_{i \alpha, j \alpha^{\prime}}(\phi)=\int_{-1}^{1} F(x) D_{i \alpha, j \alpha^{\prime}}(\phi, x) d x
$$

where

$$
D_{i \alpha, j \alpha^{\prime}}(\phi, x)=\sum_{\lambda} U_{\lambda, i \alpha}^{\dagger} U_{j \alpha^{\prime}, \lambda} \delta\left(x-\epsilon_{\lambda}\right)
$$

and $F(x)$ is a function that defines the observable in question. The quantities $U_{j \alpha, \lambda}$ and $\epsilon_{\lambda}$ denote the eigenvectors and eigenvalues of the normalized one-electron Hamiltonian, $H(\phi)$, respectively. (In general, $i$ and $j$ are spatial indices and $\alpha$ and $\alpha^{\prime}$ are indices corresponding to internal degrees of freedom if any. Spatial indices will always be denoted without arrows or bold letters independently of the dimension since on any lattice a vector $\mathbf{v}$ can be mapped into a single integer. Also the notation $i+j$ is meant to represent the lattice site given by the vectorial sum of the vectors corresponding to $i$ and $j$ respectively.) For example, in the context of a one-orbital spin-fermion model with spin, consider the observable: $\left\langle c_{i \sigma}^{\dagger} c_{j \sigma}\right\rangle=\sum_{\lambda} U_{\lambda, i \sigma}^{\dagger} U_{j \sigma, \lambda} f\left(\beta\left(a \epsilon_{\lambda}+b-\mu\right)\right)$, with $f(x)=$ $\frac{1}{\left(\mathrm{e}^{x}+1\right)}$ and the $F(x)$ of Eq. (6) is $F(x)=f(\beta(a x+b-\mu))$ for this particular observable.(The normalization factors $a$ and $b$ where defined in Eq. (11).) Now, $F(x)$ is expanded in Chebyshev polynomials, $F(x)=\sum_{m=0}^{\infty} f_{m} T_{m}(x)$, and the coefficients $f_{m}$ are calculated by inverting this formula. In all equations $\langle\ldots\rangle$ denotes the average over the fermionic sector for a fixed configuration of classical fields. The moments of $D_{i \alpha, j \alpha^{\prime}}$ are defined by:

$$
\mu_{i \alpha, j \alpha^{\prime} ; m}(\phi)=\left\langle i \alpha\left|T_{m}(H(\phi))\right| j \alpha^{\prime}\right\rangle,
$$

and therefore:

$$
A_{i \alpha, j \alpha^{\prime}}(\phi)=\sum_{m} f_{m} \mu_{i \alpha, j \alpha^{\prime} ; m}(\phi),
$$

which is the formula used for the calculation of the observable $A_{i \alpha, j \alpha^{\prime}}$ with the TPEM. The computational complexity of the calculation of $\mu_{i \alpha, j \alpha^{\prime} ; m}$ can be inferred from the computational complexity of the calculation of the moments of the density of states, $\frac{8}{,}$ and is $O(1)$ for TPEM and $O(N)$ for PEM. In general, non-local observables involve a sum over the index $i$ or $j$ in the previous equation. For instance, the kinetic energy is written as $K=-t \sum_{\langle i j\rangle, \sigma}\left(c_{i \sigma}^{\dagger} c_{j \sigma}+\right.$ H.c. $)$ and, therefore, the complexities are multiplied by a factor of $N$. However, this still gives $O(N)$ complexity when the truncated expansion is used. It is important to remind the reader that two-particle observables are not required at every change of classical fields in a Monte Carlo simulation but are computed at most only once per Monte Carlo 
step. One observable of particular interest is the spectral function, $A(k, \omega)$ that can measured with Angle Resolved Photoemission Spectroscopy (ARPES). This observable can be expressed in terms of the eigenvalues $\epsilon_{\lambda}$ and eigenvectors $U_{\lambda, i \sigma}$ of the normalized Hamiltonian ${ }^{35}$ : $A_{\phi}(r, \omega)=\sum_{i, \sigma, \lambda} U_{\lambda, i \sigma}^{\dagger}(\phi) U_{(i+r) \sigma, \lambda}(\phi) \delta\left(\omega^{\prime}-\epsilon_{\lambda}\right)$, where $\omega^{\prime}=(\omega-b) / a$ is the normalized frequency. Then, it is straightforward to show that the function $F(x)$ of Eq. (6) for $A(r, \omega)$ is $F^{A}(x)=\delta\left(\omega^{\prime}-x\right)$, which allows for the calculation of the Chebyshev coefficients in terms of the moments of $D_{i \sigma, j \sigma}(\phi, x)$ with the help of Eq. (4):

$$
A(r, \omega)=\sum_{m} \frac{\alpha_{m} T_{m}\left(\omega^{\prime}\right) \sum_{i \sigma} \mu_{i \sigma,(i+r) \sigma ; m}}{\pi \sqrt{1-\omega^{\prime 2}}} .
$$

(Note that $\alpha_{m}=2-\delta_{m, 0}$ was defined previously.)

\section{Four-particle observables}

Linear responses cannot in general be expressed in terms of only two-particle observables but they can nevertheless be calculated with the formalism of the TPEM without introducing random vectors, uncontrolled approximations or systematic errors, as will be shown. The discussion here will be focused on the optical conductivity but the procedure can be generalized to other observables as long as there is an operator that plays the role of the current. The key step is based on a recent development

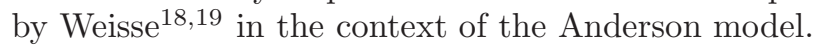

For any configuration of classical fields $\phi$, the optical conductivity ${ }^{3}$ can be rewritten as $(f(x)$ is the Fermi function $): \sigma(\omega)_{\phi}=\frac{\pi}{\omega N} \sum_{\lambda \lambda^{\prime}}\left|\left\langle\lambda\left|j_{x}\right| \lambda^{\prime}\right\rangle\right|^{2}\left(f\left(\beta\left(E_{\lambda^{\prime}}-\mu\right)\right)-\right.$ $f\left(\beta\left(E_{\lambda}-\mu\right)\right) \delta\left(\omega-E_{\lambda}+E_{\lambda^{\prime}}\right)$, where $|\lambda\rangle$ and $E_{\lambda}$ denote the eigenvectors and eigenvalues of the one-electron Hamiltonian. Note that $E_{\lambda}$ is related to the normalized Hamiltonian eigenvalues simply by $E_{\lambda}=a \epsilon_{\lambda}+b$ (see Eq. (11)). Now, following Reference 18, $\sigma(\omega)$ can be expressed in terms of the function $j_{\phi}(x, y)$ :

$$
\begin{aligned}
j_{\phi}(x, y)= & \frac{1}{N} \sum_{\lambda \lambda^{\prime}}\left|\left\langle\lambda\left|j_{x}\right| \lambda^{\prime}\right\rangle\right|^{2} \delta\left(x-\epsilon_{\lambda}\right) \delta\left(y-\epsilon_{\lambda^{\prime}}\right) \\
\sigma_{\phi}(\omega)= & \frac{1}{\omega} \int_{-\infty}^{\infty} j_{\phi}(x, x+\omega)[f(\beta(a x+b-\mu))- \\
& f(\beta(a x+b-\mu+\omega))] d x
\end{aligned}
$$

The function $j_{\phi}(x, y)$ can be expanded in Chebyshev polynomials: $j_{\phi}(x, y)=\sum_{m n} \eta_{m n}(\phi) T_{m}(x) T_{n}(x)$, with $\eta_{m n}(\phi) \equiv \operatorname{Tr}\left(T_{m}(\hat{H}(\phi)) j_{x} T_{n}(\hat{H}(\phi)) j_{x}\right) / N$. If the above trace is evaluated in the real-space basis, $\{\mid i>, \sigma\}$, of the one-electron sector, then it is possible to express $\eta_{m n}$ in terms of the coefficients $\mu_{i \sigma, j \sigma^{\prime} ; m}$ defined in Eq. (8):

$$
\begin{aligned}
\eta_{m n}(\phi)= & \sum_{i, j, k, l} \sum_{\sigma_{1}, \sigma_{2}, \sigma_{3}, \sigma_{4}} \mu_{i \sigma_{1}, j \sigma_{2} ; m}(\phi)\left\langle j \sigma_{2}\left|j_{x}\right| k \sigma_{3}\right\rangle \times \\
& \times \mu_{k \sigma 3, l \sigma 4 ; n}(\phi)\left\langle l \sigma_{4}\left|j_{x}\right| i \sigma_{1}\right\rangle
\end{aligned}
$$

and the elements $\left\langle i \sigma\left|j_{x}\right| j \sigma^{\prime}\right\rangle$ can be easily calculated: they are equal to $\pm i t e \delta_{\sigma, \sigma^{\prime}}$ if $j=i \pm \hat{x}$ or 0 otherwise. Now it is possible to calculate, $\eta_{m n}(\phi)$ for any classical field configuration $\phi$. Finally, a Monte Carlo averaging over the configurations $\phi$ is performed that yields $\sigma(\omega)$ :

$$
\begin{aligned}
\sigma(\omega)= & \frac{1}{\omega} \int_{-\infty}^{\infty} \sum_{m n}\left\langle\eta_{m n}(\phi)\right\rangle_{\phi} T_{m}(x) T_{n}(x+\omega) \times \\
& (f(\beta x)-f(\beta(x+\omega))) d x .
\end{aligned}
$$

Note that the Monte Carlo procedure has to average only $M \times M$ quantities $\left\langle\eta_{m n}(\phi)\right\rangle_{\phi}$ and the computation of $\sigma(\omega)$ using the previous equation needs to be performed only once per Monte Carlo step. It is also worth remarking that our method differs from the one proposed by Iitaka et al. ${ }^{20}$ because it does not introduce random vectors and it is applicable at finite temperature.

\section{E. Parallel Computation}

Another advantage of the TPEM, is that it can be parallelized effectively up to 50-100 processors, because the calculation of the moments for each basis ket $|\nu\rangle$ is independent. Thus, the basis can be partitioned in such a way that each processor calculates the moments corresponding to a portion of the basis. It is important to remark that the data to be moved between different nodes are small compared to calculations in each node. Indeed, the communication time is proportional to $M N_{P E}$ and communication among nodes is mainly done here to add up all the moments. The exact diagonalization algorithm can be parallelized only up to very few processors even when considering sparse matrices.

The calculation of observables that will be presented in the next section was carried out by using parallel computation. The complexity scales with the number of processors up to the size of the one-electron Hilbert space, $N_{\text {dim }}$. However, the observable that needs to be calculated more frequently is the difference in effective action. Due to the truncation in this action difference that takes place when the TPEM is used (see e.g. Ref. 8), the sum in this case is performed only up to the size of the truncated Hilbert space, $A$. This implies that the inverse complexity scales with the number of processors, $N_{P E}$ only up to $N_{P E}=A$ and not $N_{P E}=N_{\text {dim }}$. Although this imposes a limitation of the parallelization algorithm, since in general $A \ll N_{d i m}$, energy integrated observables usually converge for a cutoff $M \leq 20$ and less than $A$ processors is enough in most cases ${ }^{14}$.

However, energy dependent or many-body observables, e. g. the density-of-states and the optical conductivity, need more moments (usually 100 or more as will be discussed in the next section) for their calculation within the same error. Now, increasing the number of moments $M$ implies an increase of the truncated Hilbert space of size $A$. But even though now the number of moments has to be increased, the threshold for the scaling of the parallelization algorithm has also increased. Therefore, these 
types of problems are particularly suited to be studied efficiently with supercomputers with thousands of nodes.

\section{APPLICATION: DOUBLE EXCHANGE MODEL FOR MANGANITES}

\section{A. Microscopic Model}

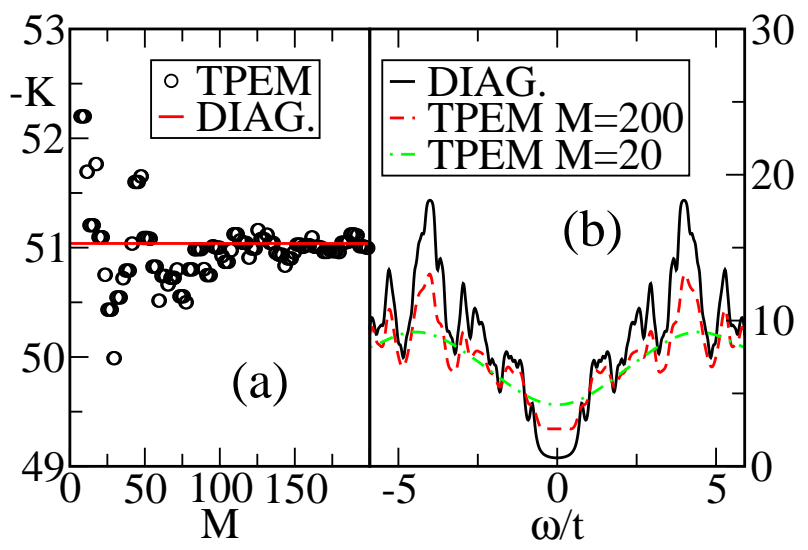

FIG. 1: (a) Kinetic energy of a single configuration of classical spins on a $8 \times 8$ lattice for model Eq. 15 calculated with the DIAG and TPEM algorithms. The configuration was obtained after evolving the model with Monte Carlo with 600 steps for $J_{H}=4 t$ and $\mu=-4 t, T=0.02 t$ and $J_{A F}=0$. $\epsilon_{p r}=10^{-5}$ and $\epsilon_{t r}=10^{-6}$. (b) Density-of-States for the model for the same configuration and parameters as in (a). Algorithms and different expansion cut-offs are indicated.

Manganese oxides, known as manganites, are correlated-electron systems of much current interest in condensed matter physics. This interest started with the discovery of the so-called "colossal" mangetoresistance (CMR) effect, an effect three orders of magnitude larger than the one observed previously on superlattice films 21

Considerable progress has been achieved in understanding many properties of these compounds by using double exchange models ${ }^{22}$ The electron-phonon coupling is usually neglected but it can and has been included; the phonon degrees of freedom are, then, an additional classical field. In the ferromagnetic phase the electrons directly jump from manganese to manganese spin and their kinetic energy is minimized when these spins are aligned. Moreover, the unbiased study of spin-fermion models for manganites as presented here can produce other long- and short-range ordered phases, e. g. antiferromagnetism, inconmensaturate phases (to be described later) and phase separation. In general, the correct ground states and very good estimates of Curie and Neél temperatures have been obtained with the DIAG method applied to double-exchange models ${ }^{2}$. The Hamiltonian of the system can be written $\operatorname{as}^{23.24}$ :

$$
\hat{H}=-t \sum_{\langle i j\rangle, \sigma} \hat{c}_{i \sigma}^{\dagger} \hat{c}_{j \sigma}-J_{H} \sum \vec{S}_{i} \cdot \vec{\sigma}_{i}+J_{A F} \sum_{\langle i j\rangle} \vec{S}_{i} \cdot \vec{S}_{j}
$$

where $\hat{c}_{i \sigma}^{\dagger}$ creates a carrier ( $e_{g}$ electron) at site $i$ with spin $\sigma$. The carrier-spin operator interacting ferromagnetically with the localized Mn-spin $\vec{S}_{i}$ is $\vec{\sigma}_{i}=$ $\sum_{\alpha, \beta} \hat{c}_{i \alpha}^{\dagger} \vec{\sigma}_{\alpha, \beta} \hat{c}_{i \beta}$. For the manganites, the Hund coupling $J_{H}$ is large compared to the hopping energy scale $t$ and, as a consequence, suppresses double occupancy. In this respect, a large $J_{H}$ coupling acts as a large Hubbard repulsion. The last term is the superexchange between localized $t_{2 g}$ spins.

Static properties of this model, e. $g$. the local spin magnetization and energy of the system have been previously studied with the TPEM on large lattices $\stackrel{14}{1}$ Here the focus will be on more complex observables. Before showing results for dynamical observables we will present first results for a two-particle static observable, namely, the kinetic energy, $K$. A simulation was performed in the ferromagnetic region of Hamiltonian Eq. (15) with $J_{H}=4 t$ and quarter filling. The kinetic energy is shown in Fig. 1] for a fixed configuration of classical fields (spins, $S_{i}$, in this case) calculated both with the diagonalization method and with the TPEM for various values of the cutoff, $M$. The configuration of classical spins was selected after evolving the system with Monte Carlo at $T=0.02 t . K$ converges to the exact value within an error window of less than $2 \%$ for $M \geq 100$ which implies that the required cutoff is larger than for one-particle or classical observables $\frac{14}{}$

Periodic boundary conditions will be used throughout the following calculations. The unit of energy is the hopping parameter, $t$.

\section{B. Density of States and Spectral Function}

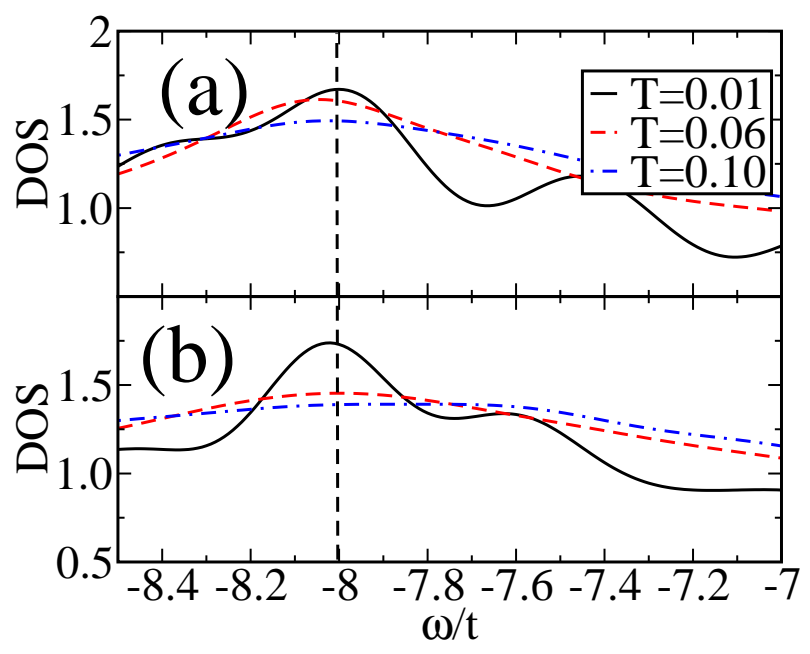

FIG. 2: DOS at three temperatures on a $20 \times 20$ lattice calculated with the TPEM and the Monte Carlo algorithm, $M=200$, for model Eq. 15 at $J_{H}=8 t,\langle n\rangle=0.5$ for (a) $J_{A F}=0$ and (b) $J_{A F}=0.02$. The location of the chemical potential is indicated by the vertical dashed line. 


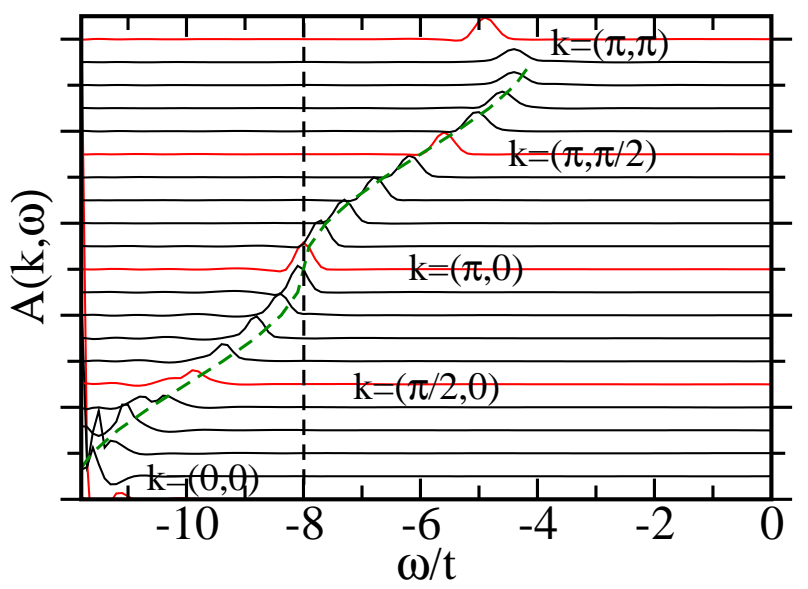

FIG. 3: Spectral function for the double exchange model calculated on a $20 \times 20$ lattice with the TPEM at $J_{H}=8 t$, $n=0.5, T=0.02 t$ (FM system). The curves are parameterized in terms of $\left(k_{x}, k_{y}\right)$ from $(0,0)$ to $(\pi, 0)$ to $(\pi, \pi)$. The location of the Fermi energy is indicated by the vertical dashed line. There is a finite DOS at the Fermi energy. The dashed line is the spectrum calculated analytically for a perfect ferromagnet.

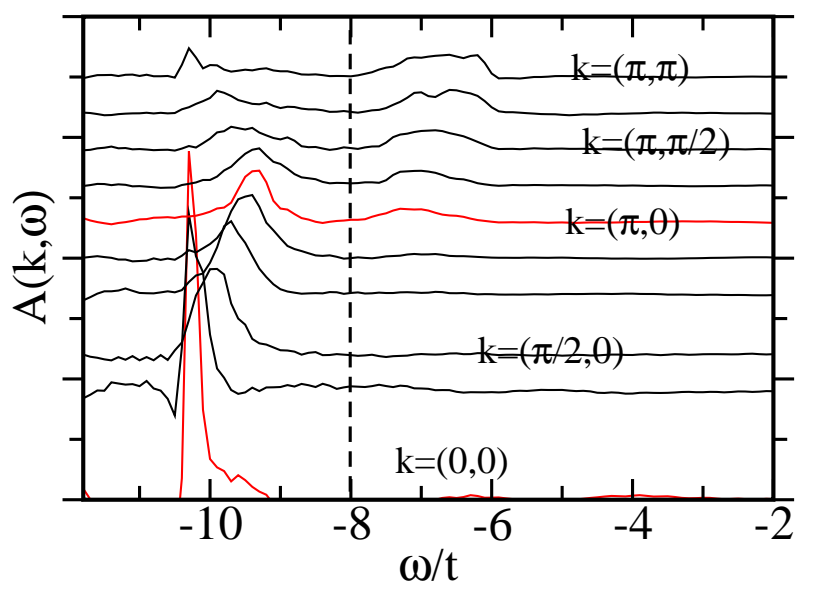

FIG. 4: For $J_{A F}=0.15$ and the same parameters as in Fig. 3 corresponding to an insulator, $A(k, \omega)$ shows a clear gap and a more complex form. The vertical dashed line indicates the position of the Fermi energy.

We will analyze Eq. (15) at quarter filling $(\langle n\rangle=0.5)$ and $J_{H}=8 t$. For $J_{A F}=0$ the ground state of the system is ferromagnetic. As $J_{A F}$ is increased the superexchange coupling competes with the double-exchange term and there exists a critical $J_{A F}$ beyond which the system's ground state is no longer ferromagnetic. This critical $J_{A F}$ was found to be $0.05 \pm 0.01$ in two dimensions and $0.12 \pm 0.01$ in one dimension. Above this value, the structure factor presents peaks at incommensurate momenta (and if $J_{A F}$ is large enough the system becomes antiferromagnetic). In the next section we will show that the ferromagnetic region is metallic, whereas the region with incommensurate momenta is insulating.
First we show that the DOS calculated with the TPEM gives reasonable results by comparing with the ones obtained with the DIAG (exact) method. This is presented in Fig. Пo for the same parameters as in Fig. П. The finite size effects of the DOS are only reproduced by the TPEM with 100 moments or more. Unlike energy integrated quantities, energy dependent quantities need more moments for convergence, as expected. The DOS in the ferromagnetic phase has two bands centered at approximately $\pm J_{H}$ each with bandwidth equal to $4 t$.

Now that we know that the TPEM yields reliable results for the DOS we proceed to perform Monte Carlo simulations. In Fig. 2] the DOS is shown for different temperatures on a $20 \times 20$ lattice at $\langle n\rangle=0.5$ and $J_{H}=8 t$. The dependence with temperature is very mild at $J_{A F}=0$ (Fig. 2a) and there is a slight decrease of the DOS at the Fermi energy for $J_{A F}=0.02$ (Fig. 2b) which is still ferromagnetic. There is no indication of a pseudogap forming at higher temperatures in the ferromagnetic region. However, in photoemission studies for manganite ${ }^{25}$ the DOS of metallic samples was found to depend more strongly on temperature and to display a more complicated structure than the one shown in Fig. 2. It is very likely that the inclusion of phonons or many orbitals are necessary to be able to reproduce these features. The influence of these corrections will be prominent mainly near the critical $J_{A F}$ that separates the metallic from the insulating regions. This suggests that the relevant regime of our model for typical ferromagnetic samples lies very near the metal-insulator transition that occurs with increasing $J_{A F}$. We will see evidence of this also when we examine the optical conductivity and conductances in the next section.

Our tests show that the calculation of the spectral function with Eq. 10 requires at least $M=100$ for this model. Using a large value for $M, A(k, \omega)$ is plotted in Fig. [3]and 4 on a $20 \times 20$ lattice for $J_{H}=8 t$ at low temperature $(T=0.02 t)$ and quarter filling. A total of 1000 thermalization steps and 1000 measurement steps were done first with $M=40$. An additional 100 steps were used to calculate $A(k, \omega)$ with a large number of moments for the expansion. For $J_{A F}=0$, Fig. [3] corresponding to a ferromagnet, there is a single peak for every value of $k$, as expected since the system is ferromagnetic. There is also a clear Fermi surface satisfying $\cos \left(k_{x}\right)+\cos \left(k_{y}\right)=0$ and the peaks follow the dispersion calculated analytically for a perfect FM. But for $J_{A F}=0.15$ in Fig. [4 $A(k, \omega)$ presents a complex form and also a gap opens at the Fermi energy. It is worth noting that the structure factor at this value of $J_{A F}$ has peaks at incommensurate momenta, e. $g \cdot\left(k_{x}, k_{y}\right)=(\pi, 0)$ that are precursors of the antiferromagnetic phase that forms at even larger $J_{A F}$.

In all cases, the moments where multiplied by damping factors to reduce the high frequency oscillations as explained before. 


\section{Optical Conductivity}

The optical conductivity in units of $e^{2} / \hbar$ is shown in Fig. [5] on a $6^{3}$ lattice for two configurations of classical spins obtained at two different temperatures. The figure shows that the TPEM with 200 moments gives results consistent with the diagonalization method. The value of $T_{C}=0.09 \pm 0.01$ was determined from the maximum distance correlation between classical spins (Fig. 6) taken as distance $=L \sqrt{3} / 2$, with $L=6$ being the linear length of the lattice. Below $T_{C}$ there is a single peak at $\omega=0$ and above $T_{C}$ there is an additional peak at $\omega=2 J_{H}$ that increases in intensity as temperature is increased. This peak at finite $\omega \approx 2 J_{H}$ is due to transitions from both the spins up and down since they have similar occupations above $T_{C}$. On the other hand, below $T_{C}$ the electronic states with spin antiparallel to the $t_{2 g}$ spins have high energies and, therefore, the transition across the $2 J_{H}$ gap weakens and only the "Drude" peak at $\omega=0$ is left.

Fig. 5 is in qualitative agreement with the optical conductivity spectra of $\mathrm{La}_{1-x} \mathrm{Sr}_{x} \mathrm{MnO}_{3}$ (see e. g. Ref. 26) where the intensity is transfered from low to high energy as temperature increases. Then, our three dimensional results suggest that the spin-fermion model considered here contains the basic physics to reproduce the behavior of the optical conductivity with temperature at least in the metallic regime. A more accurate treatment would include many orbitals, in particular if orbital ordered phases, e. $g$. the CE phase ${ }^{27}$, are relevant ${ }^{28,29}$.
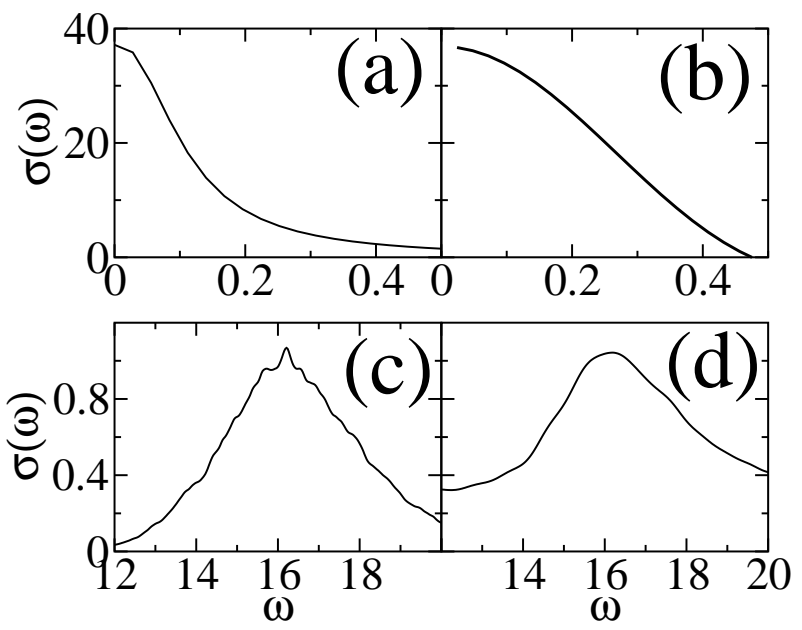

FIG. 5: Optical conductivity on a $6^{3}$ lattice calculated with the TPEM and DIAG for the same configurations of classical fields at $J_{H}=8 t$ and quarter filling. (a) $T=0.02 t$ calculated with the DIAG (b) $T=0.02 t$ calculated with the TPEM, (c) $T=0.2 t$ calculated with DIAG and (d) $T=0.2 t$ calculated with the TPEM. In (b) and (d) $M=200$.

Now the TPEM will be used to determine the metal to insulator transition that occurs in the model when a $J_{A F} \neq 0$ is considered in Eq. (15). This transition was found in one dimensional finite chains at zero temperature ${ }^{30}$. The quantity of interest is the limit of

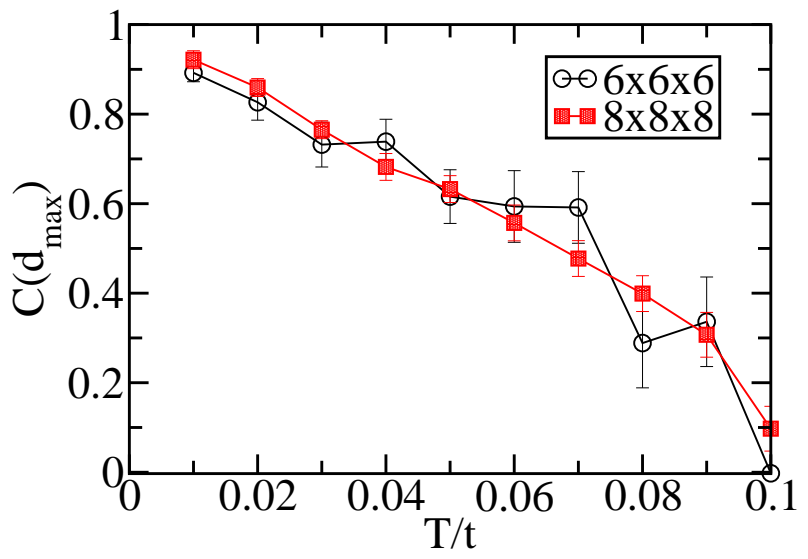

FIG. 6: Maximum distance spin-spin correlation, $C\left(d_{\max }\right)$, vs. temperature for the ferromagnetic regime $\left(J_{A F}=0\right)$ on $6^{3}$ and $8^{3}$ lattices. $d_{\max }=\sqrt{3} L / 2$ where $L=6$ or $L=8$.

$\sigma(\omega)$ as $\omega \rightarrow 0$, yielding the conductance $C$. A total of 1000 Monte Carlo steps for thermalization and 1000 measurement steps were performed with $M=40$. An additional 100 steps were used to calculate dynamical properties with a large number of moments for the expansion (usually $M=200$ ). We will examine the threedimensional case for two values of the superexchange coupling $J_{A F}$. In Fig. 7 for $J_{A F}=0$ (corresponding to a ferromagnetic ground state), $C$ is plotted as a function of temperature on $6^{3}$ and $8^{3}$ lattices with $J_{H}=8 t$ and $\mu=-8 t, i$. $e$. for a density approximately equal to quarter filling. As expected $C$ decreases monotonically with increasing temperature. On the other hand, for $J_{A F}=0.1 t$ the ground state is not ferromagnetic anymore but it is given by states with incommensurate momenta instead. These states are insulating as can be seen from Fig. 17. Therefore, the present method allows for the analysis of the metal to insulator transition with increasing $J_{A F}$ in the three dimensional model. It was essential to be able to run simulations on $6^{3}$ and $8^{3}$ lattices since $4^{3}$ is known to show strong finite size effects (e. $g$. the low temperature magnetization increases with temperature in that case). Simulations on $8^{3}$ or more lattice sites are not possible with the DIAG method.

Note that the standard dependence of the resistivity on temperature for CMR manganites cannot be reproduced with the model given by Eq. (15): the calculated resistivity has no peak at finite temperature, only a monotonous behavior. However, there is indication 31 that chemical disorder could transform an insulator into a poor metal at low temperatures. For this to happen the parameters of the system $\left(J_{A F}\right.$ in our model) have to be located very near the border of the metal-insulator separation. This is in agreement with the experimental observation ${ }^{32}$ that CMR manganites in the ferromagnetic region have poor conductance and with the results presented before for the density-of-states and spectral function and provide further evidence that this is the relevant regime to investigate for possible explanations of the CMR effect. In 
other words, if small corrections, $e . g$. disorder, many orbitals or weakly-coupled phonons, are included far from the critical $J_{A F}$, they will not affect the qualitative trends shown here but they are expected to be very important near this critical $J_{A F}$.

The Hamiltonian parameter corresponding to the superexchange interaction between $t_{2 g}$ spins, $J_{A F}$, drives the three-dimensional system from a metal to an insulator. In the phenomenological model of Eq. (15), $J_{A F}$ is an effective interaction that competes with the doubleexchange mechanism. This competition is related to the tolerance factor of the samples, or the deviation of the Mn-O-Mn angle. Therefore, calculations at different values of $J_{A F}$ can simulate the transport and magnetic properties of different compounds. For instance, in the $\operatorname{Pr}_{1-x}\left(\mathrm{Ca}_{1-y} \mathrm{Sr}_{y}\right)_{x} \mathrm{MnO}_{3}$ system ${ }^{33}$ distortions in the $\mathrm{MnO}_{6}$ octahedra weaken the double-exchange mechanism and the superexchange interaction becomes important as well as collective Jahn-Teller distortions. Even though in this work we have included only the superexchange coupling, the qualitative consequences of this competition can be seen in Fig. 7 Moreover, with the formalism presented in this work for the TPEM it will be possible to obtain linear responses of Hamiltonians that include Jahn-Teller phonons and many orbitals which is not the case with the DIAG method because the Hilbert space of these systems is at least twice as large as the one for the one-orbital model, $i$. e. the computations would be 16 times slower. The complexity of the TPEM scales linearly with the size of the one-electron sector.

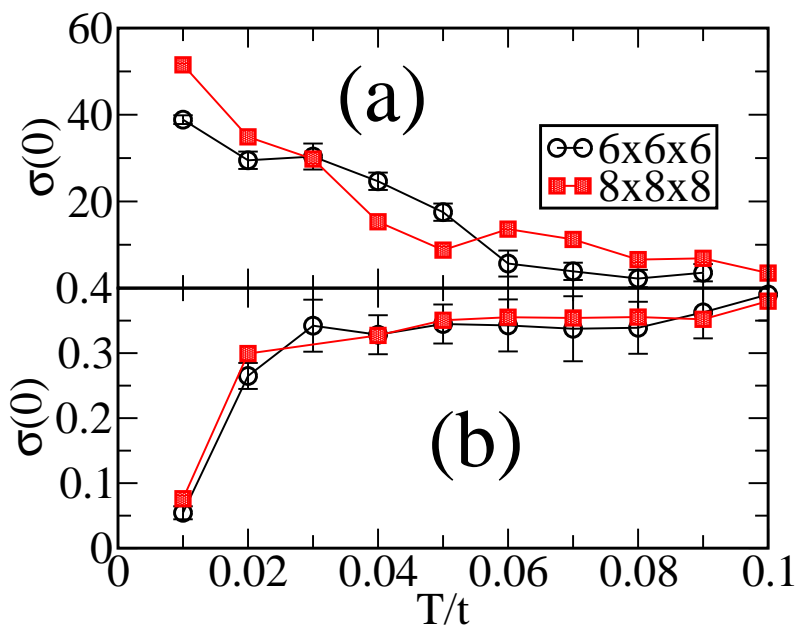

FIG. 7: $\sigma(0)$ vs. temperature at $J_{H}=8 t$ and quarter filling on $6^{3}$ and $8^{3}$ lattices for (a) $J_{A F}=0$ and (b) $J_{A F}=0.1 t$ showing metallic and insulating behavior with temperature respectively. The results were obtained with the TPEM and $M=200$.

\section{CONCLUSIONS}

The TPEM was extended to include the calculation of two- and four-particle observables without introducing systematic errors or uncontrollable approximations. By comparing with the exact algorithm it was found that energy-dependent observables need more moments than energy integrated observables but the results converge with $M \approx 100$ moments. The formulas were applied to a spin-fermion model for manganites on a $20 \times 20$ lattice to analyze the spectral function for the ferromagnetic and incommensurate phases. We concluded that the simple model of Eq. (15) cannot reproduce the DOS of typical CMR samples. The inclusion of chemical disorden or weakly-coupled phonons would be necessary and these corrections should be important near the border that separates the ferromagnetic from the region with incommensurate momenta in the phase diagram. Similar conclusions were found by studying the metal to insulator transition with increasing superexchange coupling that occurs in the three dimensional model.

The truncated polynomial expansion allowed us to calculate dynamical properties of a spin-fermion model for manganites on up to $8^{3}$ lattices without approximations. Even larger lattices will be reachable with the new generation of supercomputers that can accommodate easily thousands of nodes, as explained before in \$IE Big lattices are particularly necessary in simulations including chemical disorder. Moreover, systems with realistic band structures ${ }^{34}$ will require an increase in the size of the Hilbert space and the calculation of dynamical observables in these more realistic models will benefit from the present methods.

\section{Acknowledgments}

We would like to thank Elbio Dagotto for many suggestions to improve the presentation of this manuscript. Conversations with H. Aliaga, N. Furukawa, A Moreo, Y. Motome and I. Sergienko are also acknowledged. This research used the SPF computer program and software toolkit developed at ORNL (http://mri-fre.ornl.gov/spf). Research performed at the Oak Ridge National Laboratory, managed by UT-Battelle, LLC, for the U.S. Department of Energy under Contract DE-AC05-00OR22725.
${ }^{1}$ K. E. Schmidt and M. H. Kalos, eds., Monte Carlo Methods in Statistical Physics II (Springer Verlag, Berlin, 1984).
2 E. Dagotto, ed., Nanoscale Phase Separation and Colossal Magnetoresistance (Springer Verlag, Berlin, 2002). 
3 G. Alvarez and E. Dagotto, Phys. Rev. B. 68, 045202 (2003).

4 A. Kaminski and S. D. Sarma, Phys. Rev. Lett. 88, 247202 (2002).

5 G. Alvarez, M. Mayr, and E. Dagotto, Phys. Rev. Lett. 89, 277202 (2002).

${ }^{6}$ I. Sergienko and E. Dagotto (2005), cond-mat/0508075.

7 Y. Motome and N. Furukawa, J. Phys. Soc. Japan 68, 3853 (1999).

8 N. Furukawa and Y. Motome, J. Phys. Soc. Jpn. 73, 1482 (2004).

${ }^{9}$ H. Aliaga, Phys. Rev. B 71, 184404 (2005).

10 Y. Motome and N. Furukawa, Phys. Rev. B 68, 144432 (2003).

11 V. V. Dobrovitski and H. A. D. Raedt, Phys. Rev. E 67, 056702 (2003).

12 L. Wang, Phys. Rev. B 49, 10154 (1994).

13 J. L. Alonso, L. A. Fernández, F. Guinea, V. Laliena, and V. Martín-Mayo, Nucl. Phys. B 596, 587 (2001).

14 G. Alvarez, C. Sen, N. Furukawa, Y. Motome, and E. Dagotto, Computer Physics Communications 168, 32 (2005).

15 N. Furukawa, Y. Motome, and H. Nakata, Computer Physics Communications 142, 410 (2001).

16 R. N. Silver and H. Roeder, Int. J. Mod. Phys. C 5, 735 (1994).

17 R. N. Silver, H. Roeder, A. F. Voter, and J. D. Kress, J. Comput. Phys. 124, 115 (1996).

18 A. Weisse (2004), cond-mat/0402513.

19 A. Weisse, G. Wellein, A. Alvermann, and H. Fehske (2005), cond-mat/0504627.

20 T. Iitaka, S. Nomura, H. Hirayama, X. Zhao, Y. Aoyagi, and T. Sugano, Phys. Rev. E 56, 1222 (1997).

21 E. Dagotto, Science 309, 258 (2005).

22 E. Dagotto, T. Hotta, and A. Moreo, Physics Reports 344, 1 (2001).

23 C. Zener, Phys. Rev. 81, 440 (1952).

24 N. Furukawa, J. Phys. Soc. Japan 63, 3214 (1994).

25 D. Dessau, T. Saitoh, C.-H. Park, Z.-X. Shen, P. Villela, N. Hamada, Y. Moritomo, and Y. Tokura, Phys. Rev. Lett. 81, 192 (1998).

26 Y. Okimoto, T. Katsufuji, T. Ishikawa, A. Urushibara, T. Arima, and Y. Tokura, Phys. Rev. Lett. 75, 109 (1995).

27 H. Aliaga, D. Magnoux, A. Moreo, D. Poilblanc, S. Yunoki, and E. Dagotto, Phys. Rev. B 68, 104405 (2003).

${ }^{28}$ K. Kim, J. Jung, and T. Noh, Phys. Rev. Lett. 81, 1517 (1998).

${ }^{29}$ K. Kim, S. Lee, T. Noh, and S.-W. Cheong, Phys. Rev. Lett. 88, 167204 (2002).

30 S. Yunoki and A. Moreo, Phys. Rev. B 58, 6403 (1998).

31 C. Sen, G. Alvarez, and E. Dagotto, Phys. Rev. B 70, 064428 (2004).

32 Y. Tokura, H. Kuwahara1, Y. Moritomo, Y. Tomioka1, and A. Asamitsu, Phys. Rev. Lett. 76, 3184 (1996).

33 Y. Tomioka and Y. Tokura, Phys. Rev. B 66, 104416 (2002).

34 T. C. Schulthess and W. H. Butler, Journal of Applied Physics 89, 7021 (2001).

35 A fixed configuration of classical fields, $\phi$, is assumed. The quantities are later averaged over these classical fields by the Monte Carlo procedure explained before. The explicit dependence on $\phi$ will be omitted whenever there is no possibility of confusion. 